\title{
Chirality-Matched Catalyst-Controlled Macrocyclization Reactions
}

\author{
Jaeyeon Hwang, Brandon Q. Mercado and Scott J. Miller*
}

\begin{abstract}
Macrocycles, formally defined as compounds that contain a ring with 12 or more atoms, continue to attract great interest due to their important applications in physical, pharmacological and environmental sciences. In syntheses of macrocyclic compounds, promoting intramolecular over intermolecular reactions in the ring-closing step, is often a key challenge. Furthermore, syntheses of macrocycles with stereogenic elements confer an additional challenge, while access to such macrocycles are of great interest. Herein, we report the remarkable effect peptide-based catalysts can have in promoting efficient macrocyclization reactions. We show that the chirality of the catalyst is essential for promoting favorable, matched transition state relationships that favor macrocyclization of substrates with pre-existing stereogenic elements; curiously, the chirality of the catalyst is essential for successful reactions, even though no new stereogenic elements are created. Control experiments involving either achiral variants of the catalyst, or the enantiomeric form of the catalyst, fail to deliver the macrocycles in significant quantity in head-to-head comparisons. The generality of the phenomenon, demonstrated here with a number of substrates, stimulates analogies to enzymatic catalysts that produce naturally occurring macrocycles, presumably through related, catalyst-defined outer-sphere interactions with their acyclic substrates.
\end{abstract}




\section{Introduction}

Macrocyclic compounds are known to perform a myriad of functions in the physical and biological sciences. From cyclodextrins that mediate analyte separations, ${ }^{1}$ to porphyrin cofactors that sit in enzyme active sites, ${ }^{2}$ and to potent biologically active, macrocyclic natural products ${ }^{3}$ and synthetic variants, ${ }^{4}$ these structures underpin a wide variety of molecular functions (Figure 1a). In drug development, such compounds are highly coveted, as their conformationally restricted structures can lead to higher affinity for the desired target, and often confer additional metabolic stability. ${ }^{5}$ Accordingly, there exists an entire synthetic chemistry enterprise focused on efficient formation and functionalization of macrocycles. ${ }^{6}$

In syntheses of macrocyclic compounds, the ring closing step is often considered the most challenging step, as competing di- and oligomerization pathways must be overcome to favor intramolecular reaction. ${ }^{6 a}$ High-dilution conditions are commonly employed to favor macrocyclization of linear precursors. ${ }^{7}$ Substrate preorganization can also play a key role in overcoming otherwise high entropic barriers associated with multiple conformational states that are not suited for ring-formation. Such preorganization is most often achieved by substrate design. ${ }^{6 a}{ }^{8}$ Catalyst or reagent control that impose conformational benefits that favor ringformation are less well known. Yet, critical precedents include templating through metal-substrate complexation, ${ }^{9}$ catalysis by foldamers ${ }^{10}$ or enzymes, ${ }^{11}$ or in rare instances, by small molecules (vide infra).

Coupling macrocyclization reactions to the creation of stereogenic elements is also rare. ${ }^{12}$ Metal-mediated reactions have been applied towards stereoselective macrocyclizations wherein chiral ligands transmit stereochemical information to the products (Figure 1b). For example, atroposelective ring-closure via Heck coupling has been applied in the asymmetric total synthesis 
a
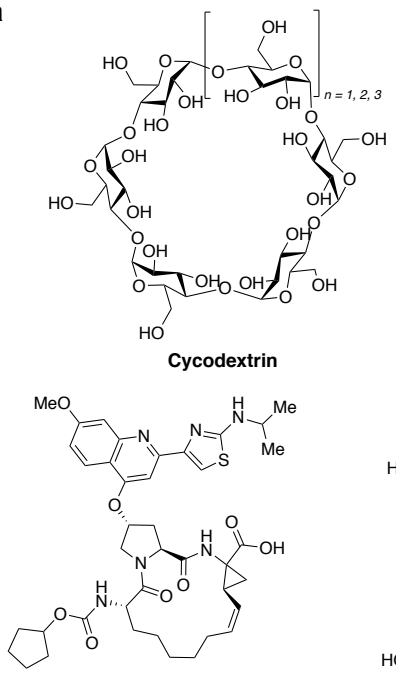

Ciluprevir
treatment of $\mathrm{HCV}$

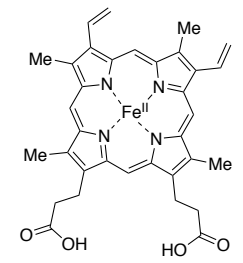

Fe-porphyrin

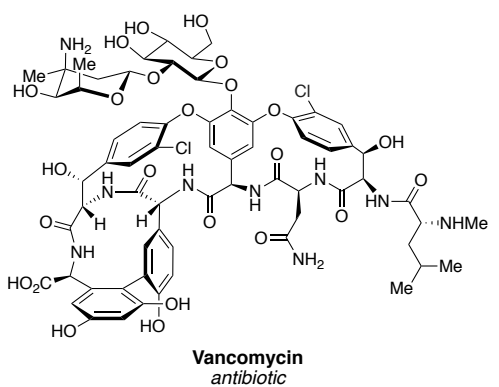

b
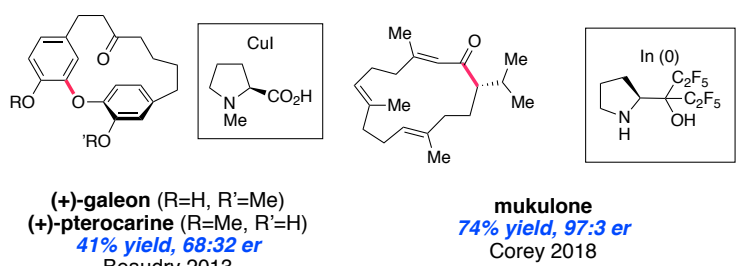

(+)-galeon $\left(\mathrm{R}=\mathrm{H}, \mathrm{R}^{\prime}=\mathrm{Me}\right)$ (+)-pterocarine ( $\left.\mathrm{R}=\mathrm{Me}, \mathrm{R}^{\prime}=\mathrm{H}\right)$ $41 \%$ yield, 68:32 er Beaudry 2013

C
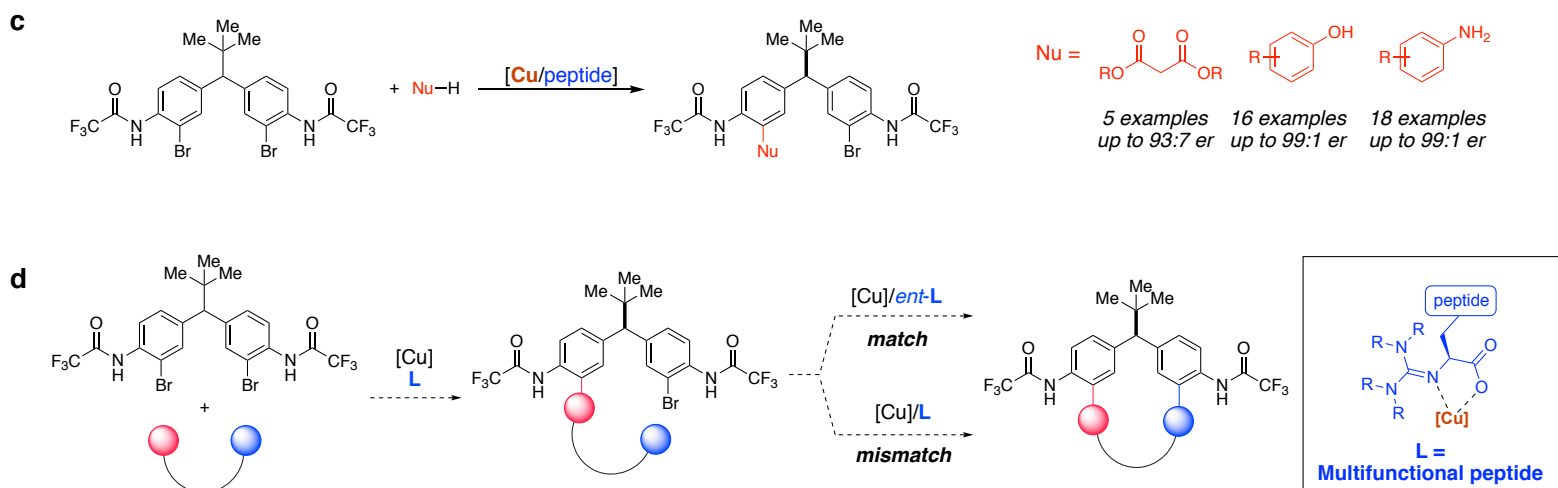

Figure 1. a) Examples of macrocyclic compounds with important applications. b) Metalmediated, catalyst-controlled stereoselective ring closures. A stereocenter or an axis of chirality is introduced at the ring closing step. Yields and er noted refer to the ring-closing step. c) Desymmetrization of diarylmethanes by $\mathrm{C}-\mathrm{C}, \mathrm{C}-\mathrm{O}$ and $\mathrm{C}-\mathrm{N}$ coupling using guanidinylated peptide ligands. d) This work: use of copper/peptidyl complexes for macrocyclization and the exploration of the matching and mismatching effect.

of Isoplagiochin D by Speichler and co-workers. ${ }^{13}$ Similarly, atroposelective syntheses of (+)galeon and other diarylether heptanoid natural products were achieved via Ullman coupling using $N$-methyl proline by Beaudry and co-workers. ${ }^{14}$ Finally, Corey and co-workers reported the enantioselective syntheses of cyclic sesquitererpenes by In-catalyzed allylation utilizing a chiral prolinol-based ligand. ${ }^{15}$ While these examples collectively illustrate the utility of chiral ligands in stereoselective macrocyclizations, such examples remain limited. 
We envisioned a different role for chiral catalysts when addressing intrinsically disfavored macrocyclization reactions. When unfavorable macrocyclization reactions are confronted, we hypothesized that outer-sphere interactions between catalyst and substrate might provide transient conformational restriction that could promote macrocyclization. To address this question, we chose to explore whether or not a chiral catalyst-controlled macrocyclization might be possible with peptidyl copper complexes. In the context of the medicinally ubiquitous diarylmethane scaffold, we had previously demonstrated the capacity for remote asymmetric induction in a series of bimolecular desymmetrizations using bifunctional, tetramethylguanidinylated (TMG) peptide ligands. For example, we showed that peptidyl copper complexes were able to differentiate between the two aryl bromides during $\mathrm{C}-\mathrm{C}, \mathrm{C}-\mathrm{O}$ and $\mathrm{C}-\mathrm{N}$ cross-coupling reactions (Figure 1c). ${ }^{16}$ Moreover, in these intermolecular desymmetrizations, a correlation between enantioselectivity and conversion was observed, revealing the catalyst's ability to perform not only enantiotopic group discrimination, but also kinetic resolution on the mono-coupled product as the reaction proceeds. ${ }^{16 \mathrm{~b}}$ This latter observation stimulated our speculation that if an internal nucleophile were present to undergo intramolecular cross-coupling to form a macrocycle, stereochemically sensitive interactions (so-called "matching" and "mismatching") ${ }^{17}$ effects could be observed (Figure 1d). Ideally, we anticipated that transition state-stabilizing interactions might even prove decisive in matched cases, the absence of catalyst-substrate stabilizing interactions might account for the absence of macrocyclization for these otherwise intrinsically unfavorable reactions. Herein, we disclose the explicit observation of these effects in chiral catalyst-controlled macrocyclization reactions. 


\section{Results \& Discussion}

Our investigation began with design of a suitable bifunctional nucleophile. A critical issue involves site-selectivity. That is, we envisioned a sequential desymmetrization-macrocyclization, wherein one reaction partner would bear two electrophilic aryl halide sites (Figure 2, 1), while the coupling partner would contain two different nucleophilic sites (Figure 2, 2a). The order of reactivity on the two nucleophilic sites of $\mathbf{2 a}$ was established through a series of competition experiments (see SI Section 7.1). For example, we found that a chemoselective coupling of diethyl malonate to 1 occurs in the presence of an unprotected aniline. In agreement with these competition experiments, 2 a underwent chemo- and enantioselective $\mathrm{C}-\mathrm{C}$ coupling using $\mathbf{L} \mathbf{1}$ to afford the desired linear precursor 3a in 66\% yield, and with a 94:6 er in analogy with our prior report (Figure 2, Eq 1). ${ }^{16 \mathrm{a}}$ This observation set the stage for evaluation of the critical diastereodifferentiating macrocyclizations. Strikingly, upon subjecting acyclic precursor 3a to Ullmann coupling conditions (see SI section 9 for optimization), using the ligand ent-L2 (enantiomeric at each stereogenic center with respect to L1), macrocycle $4 a$ was obtained in 67\% yield, and with further enantioenrichment to $>99: 1$ er (Eq 2). As is typical with $\alpha$-substituted malonates, this product is isolated as a mixture of epimerizable diastereomers due to the lability of the malonate stereogenic center. In contrast, use of $\mathbf{L} 2$ (the enantiomer of the successfully employed ent-L2) failed to deliver the macrocycle in good yield, delivering $\mathbf{4 a}$ in only $10 \%$ yield, and in racemic form. In fact, what macrocyclization is observed using the mismatched ligand $\mathbf{L} 2$ appears to be the result of the processing of the residual minor enantiomer (which is matched to L2), as evidenced by the recovery of enantiopure, unreacted starting material 3a after the macrocyclization (see SI section 8.5, page S87). Furthermore, in a striking control experiment, the achiral guanidinylated ligand $\mathbf{L 3}$ also performed poorly and quite similarly to the mismatched $\mathbf{L 2}$. These results highlight the critical 
nature of the stereochemically matched ligand for successful cyclization, and also point strongly to stabilizing cooperative effects between the right chiral catalyst and its matched substrate during the macrocyclization step. Notably, in the matched case, the macrocyclization proceeds at $45^{\circ} \mathrm{C}$ and under surprisingly typical concentrations for many bimolecular reactions $(125 \mathrm{mM})$; extreme dilution, as is typical for many macrocyclizations $(\sim 1 \mathrm{mM})$, was not required. ${ }^{6 a, 7}$
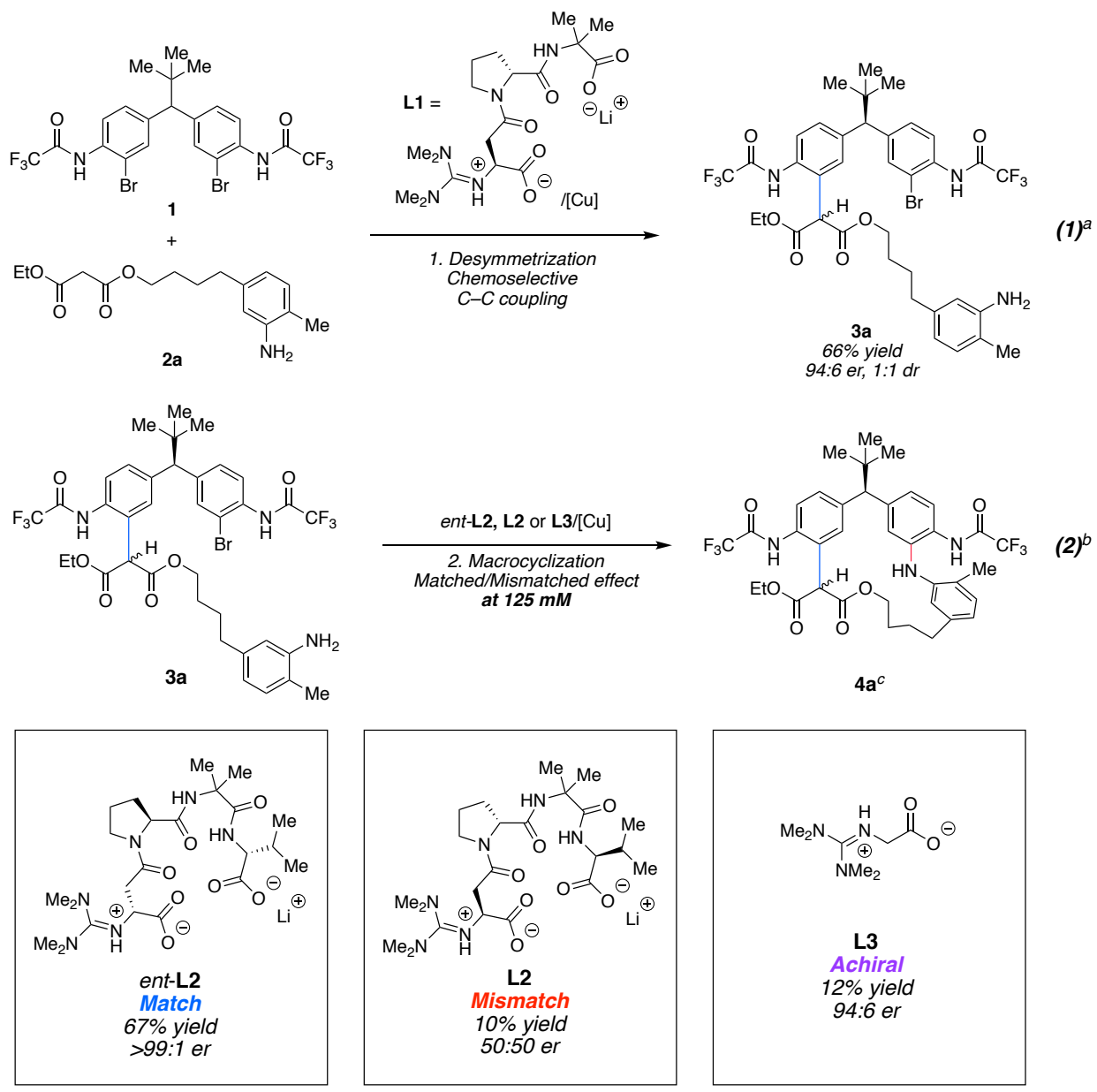

Figure 2. Chemo- and enantioselective $\mathrm{C}-\mathrm{C}$ coupling followed by diastereodifferentiating macrocyclization by $\mathrm{C}-\mathrm{N}$ coupling. ${ }^{a}$ Reaction conditions $1: 1$ (1.8 mmol, 1.0 equiv), 2a (1.0 equiv), $\mathrm{Cu}(\mathrm{MeCN})_{4} \mathrm{BF}_{4}(5 \mathrm{~mol} \%), \mathbf{L 1}(10 \mathrm{~mol} \%), \mathrm{Cs}_{2} \mathrm{CO}_{3}$ (4.2 equiv), 1:2 DMF/Tol (0.25 M), rt, $15 \mathrm{~h} .{ }^{b}$ Reaction conditions $2: 3 \mathrm{a}(0.15 \mathrm{mmol}, 1.0$ equiv), $\mathrm{CuBr}(20 \mathrm{~mol} \%)$, ligand (40 $\mathrm{mol} \%)$, $\mathrm{K}_{3} \mathrm{PO}_{4}$ (4.4 equiv), 1:2 DMF/MeCN $(0.125 \mathrm{M}), 45^{\circ} \mathrm{C}, 15$ h. ${ }^{c} 1.8: 1 \mathrm{dr}$ in $\mathrm{CD}_{2} \mathrm{Cl}_{2}$. Dr of $4 \mathbf{a}$ varies in different solvent, concentration and temperature due to the lability of the $\alpha$-malonate proton.

Encouraged by the above results, linear precursors 3a-3f were prepared to explore the scope of the reaction (Figure 3a; see SI section 4 and 6 for synthesis of 2a-2f). As with 2a, each 
substrate underwent chemo- and enantioselective $\mathrm{C}-\mathrm{C}$ coupling to yield 3a-3f in 59-69\% yield, and with enantioselectivities ranging from 94:6 er to 90:10 er (Figure 3a), setting the stage for evaluation of the generality of the chirality-matched catalyst-controlled macrocyclization reactions. In each case, the special significance of the stereochemically matched ligand was evident. Moreover, in these successful macrocyclization, further enantioenrichment was observed in the products, while the mismatched and achiral ligands consistently gave poor results (Figure 3b). For example, as illustrated in Figure 2, macrocyclization of 3a leads to the formation of 18-membered ring (4a) in 67\% yield in excellent er (>99:1 er) using ent-L2 (Figure 3b). An ortho substituent is not necessary for the demonstration of ligand effect as seen in $\mathbf{3 b}$ ( $50 \%$ yield, $>99: 1 \mathrm{er}$ ). However, the presence of an ortho substituent appears to aid in improving the yield of macrocyclized products, possibly by favoring intramolecular reaction over oligomerization pathways (3a and 3c). The effect of the mismatched ligand was less apparent for $\mathbf{3 d}$, which appear to be inherently more reactive - nevertheless, the matched ligand still dramatically outperformed the mismatched or the achiral ligands for this substrate. Linear precursor containing five methylene linkers (3e) also underwent macrocyclization. In this case, a ten-fold difference in the yield of macrocyclized product (4e) was observed, as ent-L2 afforded the product in 23\% yield (98:2 er), while $\mathbf{L} \mathbf{2}$ leads to formation of the product in only $2.5 \%$ yield ( $32: 68$ er), which once again clearly demonstrates the primacy of the matched ligand in the macrocyclization. 

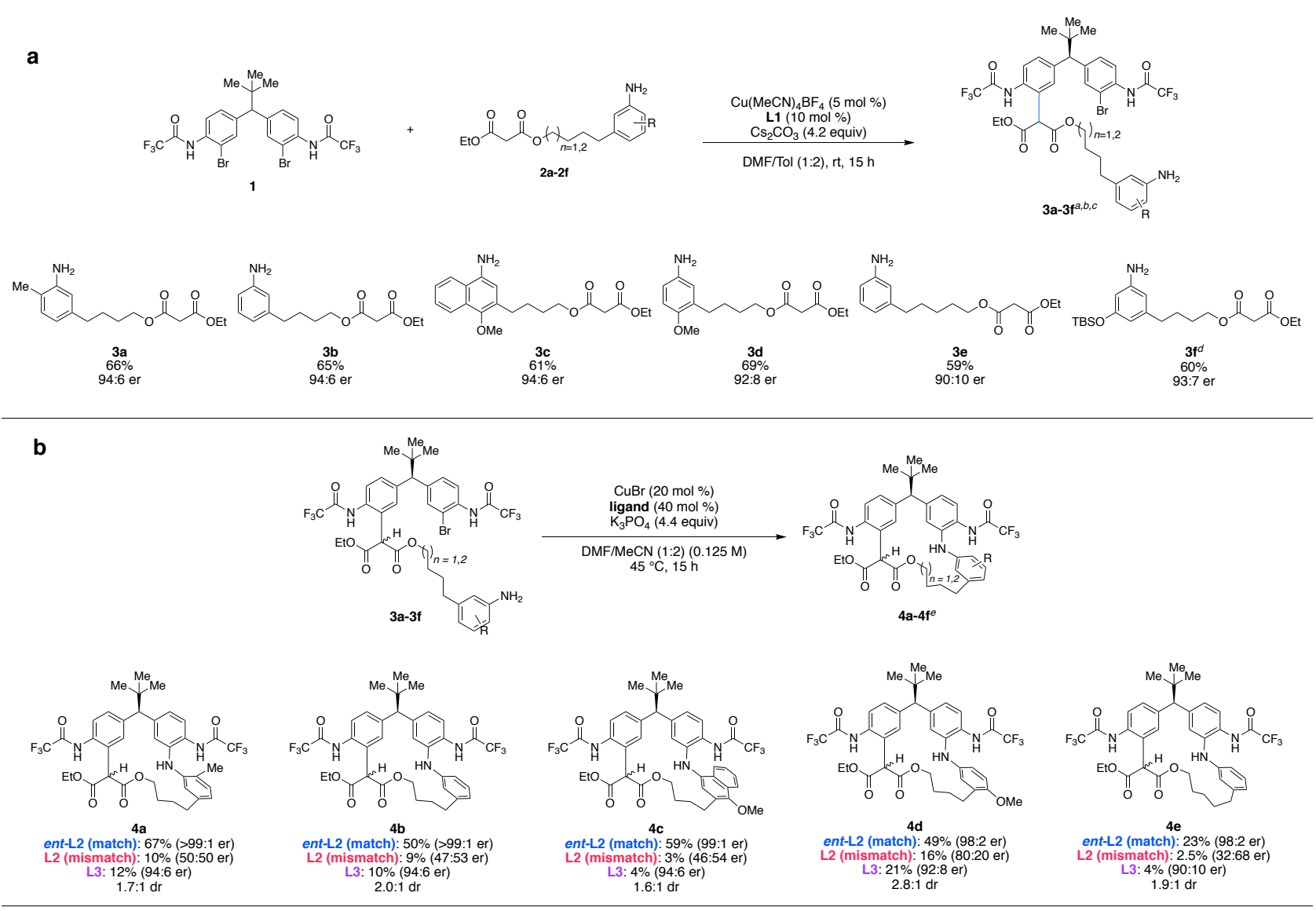

C

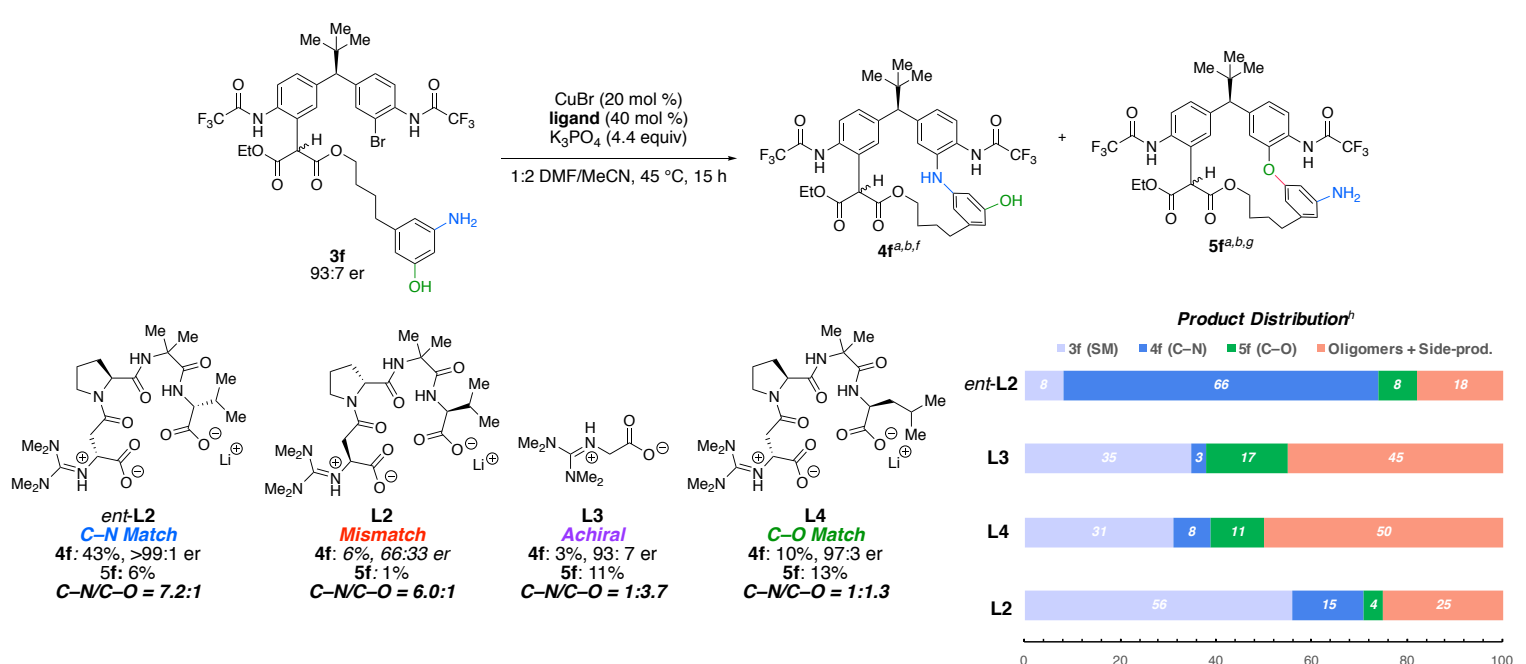

Figure 3. a) Preparation of linear precursors by chemo- and enantio-selective $\mathrm{C}-\mathrm{C}$ coupling. ${ }^{a}$ Isolated yields. ${ }^{b}$ Enantiomeric ratios were determined using chiral HPLC analysis. ${ }^{c}$ Products 3a-3f are isolated in 1.0:1 dr. ${ }^{d}$ In preparation of 3f, TBS group was removed after $\mathrm{C}-\mathrm{C}$ coupling step. See SI section 8.3 for details. b) Macrocyclization of 3a-3f: matching and mismatching effect. ${ }^{e}$ Diastereomeric ratio was determined by ${ }^{1} \mathrm{H} \mathrm{NMR}$ in $\mathrm{CD}_{2} \mathrm{Cl}_{2}$ at $25^{\circ} \mathrm{C}$. Dr's are solvent and temperature dependent due to the dynamic nature of the malonate stereocenter. c) Macrocyclization of aminophenol $\mathbf{3 f}$ using different ligands ${ }^{f_{\mathbf{4}}} \mathbf{f}$ is observed as a $2.5: 1$ mixture of diastereomers at $25{ }^{\circ} \mathrm{C}$ in $\mathrm{CD}_{2} \mathrm{Cl}_{2}$ by ${ }^{1} \mathrm{H}$ NMR. ${ }^{g} \mathbf{5}$ is observed as a $4.3: 1$ mixture of diastereomers at $25{ }^{\circ} \mathrm{C}$ in $\mathrm{CD}_{2} \mathrm{Cl}_{2}$ by ${ }^{1} \mathrm{H} \mathrm{NMR}$. ${ }^{h}$ Product distribution of macrocyclization of $\mathbf{3 f}$ based on uncorrected peak integration on UPLC-MS peaks. See SI section 12 for UPLC-MS traces. 
Strikingly, a free phenol in the substrate is tolerated, as $\mathbf{3 f}$ is processed to $\mathbf{4 f}$ in $43 \%$ yield and with 99:1 er via the preferential intramolecular $\mathrm{C}-\mathrm{N}$ coupling of $\mathbf{3 f}$ using ent-L2(C-N, $\mathbf{4 f} / \mathrm{C}-$ $\mathrm{O}, \mathbf{5 f}=7.2: 1$; Figure 3c). A particularly interesting observation is that the use of achiral ligand $\mathbf{L 3}$ actually favored macrocyclization via $\mathrm{C}-\mathrm{O}$ coupling $(\mathbf{5 f}, 11 \%$ yield), and only gave the desired $\mathrm{C}-$ $\mathrm{N}$ linked macrocycle $\mathbf{4 f}$ in $3 \%$ yield. We then explored whether we can overturn the observed selectivity by using a different guanidinylated ligand. Unfortunately, the use of a ligand previously optimized for $\mathrm{C}-\mathrm{O}$ coupling, $\mathbf{L} 4$ (entry 4 ) ${ }^{16 \mathrm{~b}}$ did not lead to efficient macrocyclization, through either $\mathrm{C}-\mathrm{O}$ coupling nor $\mathrm{C}-\mathrm{N}$ coupling, and only favored intramolecular $\mathrm{C}-\mathrm{O}$ coupling by a slight margin (1:1.3 ratio). Consistent with previous examples (Figure 3b), the use of mismatched ligand L2 is far less efficient in catalyzing macrocyclization. We speculate that in each case of an unfavorable reaction, oligomerized side-products appears at the heart of inefficiency.

We then turned our attention to structural features of these new macrocycles. Notably, these large rings exhibit stereodynamic properties. Two sets of peaks are observed by ${ }^{1} \mathrm{H}$ NMR spectroscopy, with the ratio of two apparent diastereomers, $(S, S)-\mathbf{- 4 a}$ and $(S, R)-\mathbf{4 a}$, varying as a function of different NMR solvents (see SI section 14.3). While various phenomena might account for this observation (atropisomerism and conformational isomerism), ${ }^{14,18,19,20}$ we speculated instead that this phenomena is due to slow epimerization of the malonate stereocenter via ketoenol tautomerization. To confirm the nature of the observed isomers, we performed a variabletemperature (VT) ${ }^{1} \mathrm{H}$ NMR and deuterium exchange experiments. First, while complete coalescence of resonances was not observed at temperatures of up to $120^{\circ} \mathrm{C}$ in DMSO- $d_{6}$, a change in the ratio of certain peaks is observed (Figure 4a). Second, complete deuterium exchange in methanol- $d_{4}$ is observed, but requires 2 hours to reach completion (Figure 4b), suggesting the presence of two isomers that represent epimerizing diastereomers through keto-enol 
tautomerization. The connectivity and the absolute stereochemistry of these macrocycles were unambiguously determined by X-Ray crystallography (Figure 4c).

a)
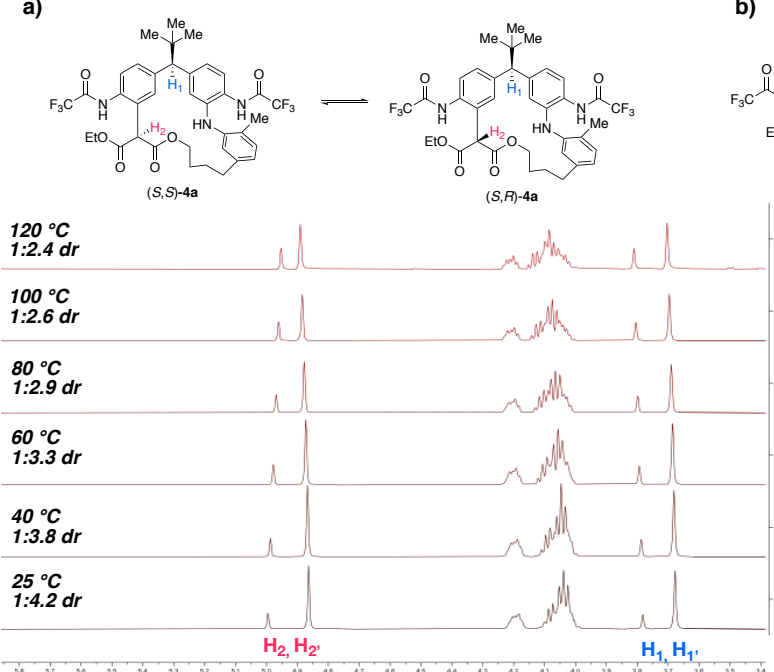

c)

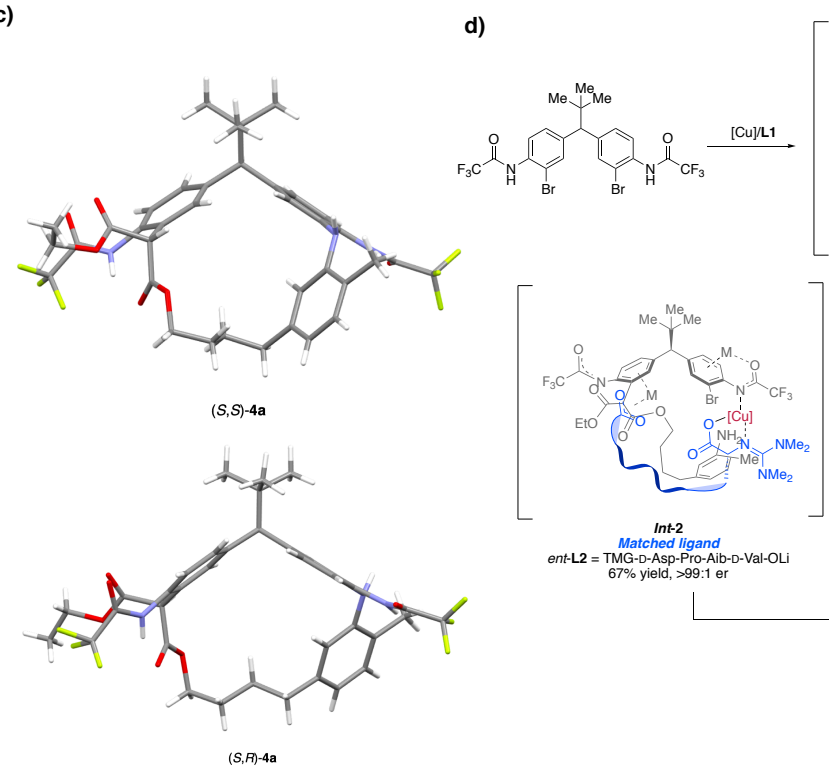

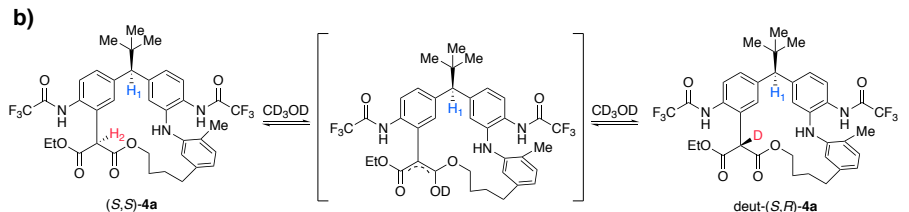

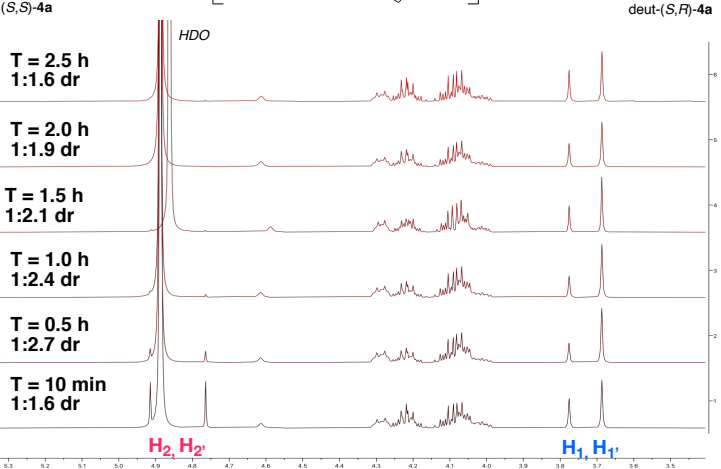

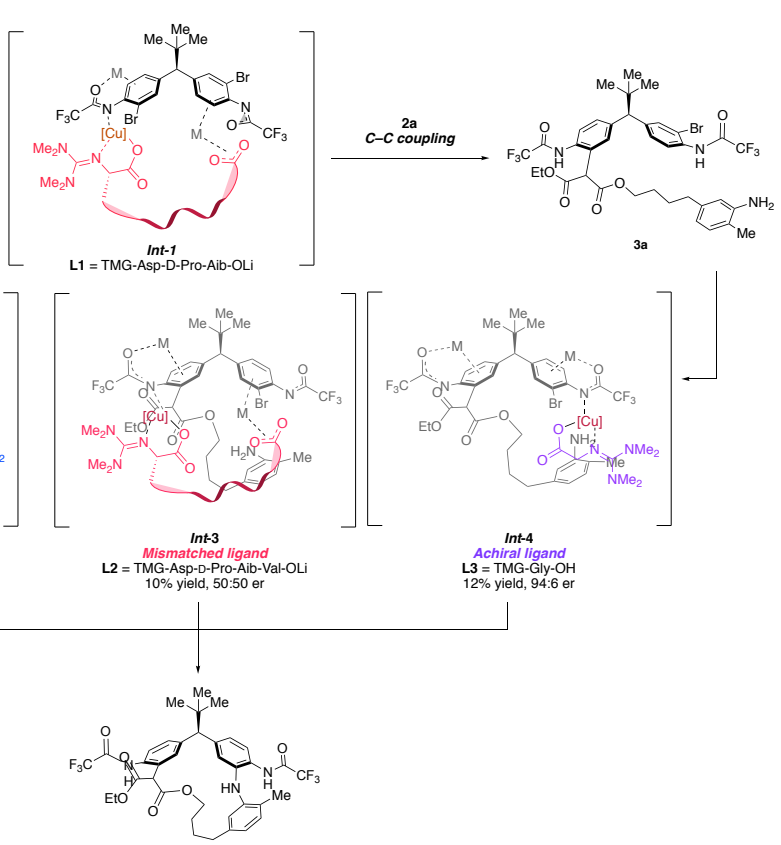

Figure 4. a) Dynamic stereochemical properties of macrocyclic diarylmethane. VT-NMR of 4a in DMSO-d ${ }_{6}(25-120$ $\left.{ }^{\circ} \mathrm{C}\right)$. b) Deuterium incorporation of $\mathbf{4 a}$ in methanol- $\mathrm{d}_{4}$ c) Crystal structure of $(S, S)-\mathbf{4 a}$ and $(S, R)-\mathbf{4 a}$, revealing the presence of both malonate epimers in the unit cell. d) Proposed model for stereochemical matching and mismatching effect in macrocyclization of diarylmethanes.

Finally, the basis of the observed stereochemical matching and mismatching in macrocyclization remains of interest. We posit that the following scenario enables chirality matched macrocyclization with peptidyl complexes (Figure 4d): first, $\mathrm{C}-\mathrm{C}$ coupling occurs on one 
aryl bromide of diarylmethane $\mathbf{1}$ using $\mathbf{L 1}$ to afford the linear precursor 3a. Then, the matched ligand (ent-L2), which is enantiomeric at the $i$ (Asp residue) and the $i+1$ (Pro residue) positions compared to $\mathbf{L 1}$, is able to efficiently catalyze macrocyclization by localizing the copper in close proximity to the aryl ring that bears the second substituting bromide (Figure $4 \mathrm{~d}$, Int-2). On the other hand, the use of the mismatched ligand, L2, leads to a sluggish reaction, as its intrinsic chirality preference is to localize the copper center in proximity to the ring that already underwent $\mathrm{C}-\mathrm{C}$ coupling, and which therefore no longer bears a bromide atom (Int-3). Presumably, the stereogenic centers of the matched catalyst are ultimately responsible for the low-energy conformations associated with the facile macrocyclization transitions state. In contrast, the mismatched ligand would then lead to a higher energy transitions state that likely suffers from unfavorable, stereochemically dictated interactions. Finally, in case of the achiral ligand $\mathbf{L 3}$, the lack of additional interaction between the carboxylate "tail" of the peptide ligand and the aryl ring may be contributing to a lower efficiency in macrocyclization (Int-4).

\section{Conclusions}

Chiral catalysts are generally employed to mediate reactions that create stereogenic elements. We have shown here that they can play a decisive role in macrocyclization reactions wherein no new element of chirality is actually formed; that is, stereochemical issues exist in transition states emanating from substrates with pre-existing chirality, and prove decisive for bond formation. Pragmatically, chiral catalysts can thus render efficient macrocyclization reactions that might otherwise not occur, or might only occur under conditions of extremely high dilution. Fundamentally, the reported observations reveal a capacity of chiral catalysts to manage highly unfavorable ring-formations through management of otherwise unfavorable entropy. That these 
observations are now recorded in a scaffold of venerable interest to medicinal chemists suggests this approach may prove broadly significant. 


\section{References}

1. Szente, L.; Szemán, J. Cyclodextrins in Analytical Chemistry: Host-Guest Type Molecular Recognition. Anal. Chem. 85, 8024-8030 (2013).

2. a) Caughey, W. S. Porphyrin Proteins and Enzymes. Annu. Rev. Biochem. 36, 611-44 (1967); b) Smith, K. M. Porphyrins. in Comprehensive Coordination Chemistry II: Volume 2 (ed. McCleverty, J. A.; Meyer, T. J.) 493-506 (Pergamon: Oxford, 2003)

3. Newman, D. J.; Cragg, G. M. Bioactive Macrocycles from Nature. In Macrocycles in Drug Discovery 1-36 (The Royal Society of Chemistry, 2015)

4. a) Vendeville, S.; Cummings, M. D. Synthetic Macrocycles in Small-Molecule Drug Discovery. In Annual Reports in Medicinal Chemistry: Volume 48 (ed. Desai, M. C.) 371-386 (. Academic Press: 2013); b) Marsault, E.; Peterson, M. L. Macrocycles Are Great Cycles: Applications, Opportunities, and Challenges of Synthetic Macrocycles in Drug Discovery. J. Med. Chem. 54, 1961-2004 (2011).

5. a) Driggers, E. M.; Hale, S. P.; Lee, J.; Terrett, N. K. The exploration of macrocycles for drug discovery - an underexploited structural class. Nat. Rev. Drug Discov. 7, 608-624 (2008); b) Giordanetto, F.; Kihlberg, J. Macrocyclic Drugs and Clinical Candidates: What Can Medicinal Chemists Learn from Their Properties? J. Med. Chem. 57, 278-285 (2014); c) Villar, E. A.; Beglov, D.; Chennamadhavuni, S.; Porco, J. A., Jr.; Kozakov, D.; Vajda, S. W., A. How proteins bind macrocycles. . Nat. Chem. Biol. 10, 723-731 (2014); d) Vinogradov, A. A.; Yin, Y; Suga, H. Macrocyclic Peptides as Drug Candidates: Recent Progress and Remaining Challenges. J. Am. Chem. Soc. 141, 4167-4181 (2019); e) Mallinson, J.; Collins, I. Macrocycles in new drug discovery. Future Med. Chem. 4, 1409-1438 (2012); f) Li, X.; Chen, S.; Zhang, W.-D.; Hu, H.G. Stapled Helical Peptides Bearing Different Anchoring Residues. Chem. Rev. 120, 10079-10144 (2020).

6. a) Martí-Centelles, V.; Pandey, M. D.; Burguete, M. I.; Luis, S. V. Macrocyclization Reactions: The Importance of Conformational, Configurational, and Template-Induced Preorganization. Chem. Rev. 115, 8736-8834 (2015); b) White, C. J.; Yudin, A. K. Contemporary strategies for peptide macrocyclization. Nat. Chem. 3, 509-524 (2011).

7. Rossa, L.; Vögtle, F. Synthesis of Medio- And Macrocyclic Compounds By High Dilution Principle Techniques. In Cyclophanes I. Topics in Current Chemistry: Vol. 113 (ed. Vögtle, F.) 3-76 (Springer: Berlin, Heidelberg, 1983).

8. a) Miller, S. J.; Blackwell, H. E.; Grubbs, R. H. Application of Ring-Closing Metathesis to the Synthesis of Rigidified Amino Acids and Peptides. J. Am. Chem. Soc. 118, 9606-9614 (1996); b) Blankenstein, J.; Zhu, J. Conformation-Directed Macrocyclization Reactions. Eur. J. Org. Chem. 2005, 1949-1964 (2005); c) Adebomi, V.; Cohen, R. D.; Wills, R.; Chavers, H. A. H.; Martin, G. E.; Raj, M. CyClick Chemistry for the Synthesis of Cyclic Peptides. Angew. Chem. Int. Ed. 58, 19073-19080 (2019).

9. a) Higman, C. S.; Nascimento, D. L.; Ireland, B. J.; Audörsch, S.; Bailey, G. A.; McDonald, R.; Fogg, D. E. Chelate-Assisted Ring-Closing Metathesis: A Strategy for Accelerating Macrocyclization at Ambient Temperatures. J. Am. Chem. Soc. 140, 1604-1607 (2018); b) Yu, M.; Wang, C.; Kyle, A. F.; Jakubec, P.; Dixon, D. J.; Schrock, R. R.; Hoveyda, A. H. Synthesis of macrocyclic natural products by catalyst-controlled stereoselective ring-closing metathesis. Nature 479, 88-93 (2011).

10. Girvin, Z. C.; Andrews, M. K.; Liu, X.; Gellman, S. H. Foldamer-templated catalysis of macrocycle formation. Science 366, 1528-1531 (2019). 
11. a) Czekster, C. M.; Ludewig, H.; McMahon, S. A.; Naismith, J. H. Characterization of a dual function macrocyclase enables design and use of efficient macrocyclization substrates. Nat. Commun. 8, 1045-1055 (2017); b) Zhang, C.; Dai, P.; Spokoyny, A. M.; Pentelute, B. L. EnzymeCatalyzed Macrocyclization of Long Unprotected Peptides. Org. Lett. 16, 3652-3655 (2014); c) Ongpipattanakul, C.; Nair, S. K. Biosynthetic Proteases That Catalyze the Macrocyclization of Ribosomally Synthesized Linear Peptides. Biochemistry 57, 3201-3209 (2018); d) Ganon, C.; Godin, É.; Minozzi, C.; Sosoe, J.; Pochet, C.; Collins, S. K. Biocatalytic synthesis of planar chiral macrocycles. Science 367, 917-921 (2020).

12. Zheng, K.; Hong, R. Stereoconfining macrocyclizations in the total synthesis of natural products. Nat. Prod. Rep. 36, 1546-1575 (2019).

13. Groh, M.; Meidlinger, D.; Bringmann, G.; Speicher, A. Atroposelective Heck Macrocyclization: Enantioselective Synthesis of Bis(bibenzylic) Natural Products. Org. Lett. 14, 4548-4551 (2012).

14. Salih, M. Q.; Beaudry, C. M. Enantioselective Ullmann Ether Couplings: Syntheses of (-)Myricatomentogenin, (-)-Jugcathanin, (+)-Galeon, and (+)-Pterocarine. Org. Lett. 15, 4540-4543 (2013).

15. Reddy, D. S.; Corey, E. J. Enantioselective Conversion of Oligoprenol Derivatives to Macrocycles in the Germacrene, Cembrene, and 18-Membered Cyclic Sesterterpene Series. J. Am. Chem. Soc. 140, 16909-16913 (2018).

16. a) Kim, B.; Chinn, A. J.; Fandrick, D. R.; Senanayake, C. H.; Singer, R. A.; Miller, S. J. Distal Stereocontrol Using Guanidinylated Peptides as Multifunctional Ligands: Desymmetrization of Diarylmethanes via Ullman Cross-Coupling. J. Am. Chem. Soc. 138, 79397945 (2016); b) Chinn, A. J.; Kim, B.; Kwon, Y.; Miller, S. J. Enantioselective Intermolecular C-O Bond Formation in the Desymmetrization of Diarylmethines Employing a Guanidinylated Peptide-Based Catalyst. J. Am. Chem. Soc. 139, 18107-18114 (2017); c) Kwon, Y.; Chinn, A. J.; Kim, B.; Miller, S. J. Divergent Control of Point and Axial Stereogenicity: Catalytic Enantioselective $\mathrm{C}-\mathrm{N}$ Bond-Forming Cross-Coupling and Catalyst-Controlled Atroposelective Cyclodehydration. Angew. Chem. Int. Ed. 57, 6251-6255 (2018).

17. Masamune, S.; Choy, W.; Petersen, J. S.; Sita, L. R. Double Asymmetric Synthesis and a New Strategy for Stereochemical Control in Organic Synthesis. Angew. Chem. Int. Ed. Engl. 24, 1-30 (1985).

18. a) Kumarasamy, E.; Raghunathan, R.; Sibi, M. P.; Sivaguru, J. Nonbiaryl and Heterobiaryl Atropisomers: Molecular Templates with Promise for Atropselective Chemical Transformations. Chem. Rev. 115, 11239-11300 (2015); b) Gasparro, F. P.; Kolodny, N. H. NMR determination of the rotational barrier in $N, N$-dimethylacetamide. A physical chemistry experiment. $J$. Chem. Ed. 54, 258 (1977); c) Eguchi, T.; Kondo, K.; Kakinuma, K.; Uekusa, H.; Ohashi, Y.; Mizoue, K.; Qiao, Y.-F. Unique Solvent-Dependent Atropisomerism of a Novel Cytotoxic Naphthoxanthene Antibiotic FD-594. J. Org. Chem. 64, 5371-5376 (1999).

19. a) Singh, S. B.; Jayasuriya, H.; Salituro, G. M.; Zink, D. L.; Shafiee, A.; Heimbuch, B.; Silverman, K. C.; Lingham, R. B.; Genilloud, O.; Teran, A.; Vilella, D.; Felock, P.; Hazuda, D. The Complestatins as HIV-1 Integrase Inhibitors. Efficient Isolation, Structure Elucidation, and Inhibitory Activities of Isocomplestatin, Chloropeptin I, New Complestatins, A and B, and AcidHydrolysis Products of Chloropeptin I. J. Nat. Prod. 64, 874-882 (2001); b) Schafer, M. S., T. R.; Sheldrick, G. M. Crystal structure of vancomycin. Structure 4, 1509-1515 (1996); c) Boger, D. L.; Weng, J.-H.; Miyazaki, S.; McAtee, J. J.; Castle, S. L.; Kim, S. H.; Mori, Y.; Rogel, O.; Strittmatter, H.; Jin, Q. Thermal Atropisomerism of Teicoplanin Aglycon Derivatives: Preparation 
of the $P, P, P$ and $M, P, P$ Atropisomers of the Teicoplanin Aglycon via Selective Equilibration of the DE Ring System. J. Am. Chem. Soc. 122, 10047-10055 (2000); d) Glunz, P. W.; Mueller, L.; Cheney, D. L.; Ladziata, V.; Zou, Y.; Wurtz, N. R.; Wei, A.; Wong, P. C.; Wexler, R. R.; Priestley, E. S. Atropisomer Control in Macrocyclic Factor VIIa Inhibitors. J. Med. Chem. 59, 4007-4018 (2016).

20. Costil, R.; Sterling, A. J.; Duarte, F.; Clayden, J. Atropisomerism in Diarylamines: Structural Requirements and Mechanisms of Conformational Interconversion. Angew. Chem. Int. Ed. 59, 18670-18678 (2020).

\section{Acknolwedgements}

This work was supported by the National Institute of General Medical Sciences (NIH grant R35 GM132092 to S.J.M.). We would like to thank Dr. A. J. Chinn, Dr. B. Kim and Dr. Y. Kwon for their advice and materials. We would also like to thank Dr. E. Paulson for assistance with NMR spectroscopy and Dr. F. Menges for assistance with mass spectroscopy.

Author Contributions J.H. and S.J.M. conceptualized the project, designed the experiments, interpreted the results. J.H. performed experiments. B.Q.M. solved and interpreted the X-ray data culminating in structure 4a. J.H. and S. J. M. prepared the manuscript with contributions from all authors; all authors contributed to discussions.

Competing Interests The authors declare no competing interests.

Materials \& Correspondence should be addressed to S.J.M. 